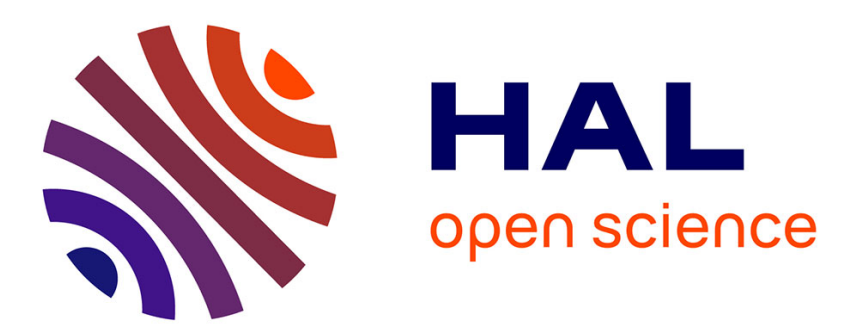

\title{
Molecules with two electronic energy levels: coupling between the molecules in the solid state via the optical and acoustic phonon branches
}

\author{
J.A. Nasser
}

\section{To cite this version:}

J.A. Nasser. Molecules with two electronic energy levels: coupling between the molecules in the solid state via the optical and acoustic phonon branches. 2018. hal-01777925

\section{HAL Id: hal-01777925 \\ https://hal.science/hal-01777925}

Preprint submitted on 25 Apr 2018

HAL is a multi-disciplinary open access archive for the deposit and dissemination of scientific research documents, whether they are published or not. The documents may come from teaching and research institutions in France or abroad, or from public or private research centers.
L'archive ouverte pluridisciplinaire HAL, est destinée au dépôt et à la diffusion de documents scientifiques de niveau recherche, publiés ou non, émanant des établissements d'enseignement et de recherche français ou étrangers, des laboratoires publics ou privés.

\section{(c)(1)}

Distributed under a Creative Commons Attribution| 4.0 International License 


\title{
Molecules with two electronic energy levels: coupling between the molecules in the solid state via the optical and acoustic phonon branches
}

\author{
Jamil A. Nasser \\ Laboratoire d'Ingénierie des Systèmes de Versailles (LISV), \\ EA 4048, CNRS, Université de Versailles Saint Quentin, \\ 45, avenue des Etats-Unis, 78035 Versailles, France and
}

In the adiabatic approximation the values of the spring constants of the springs contained in a molecule depend on its electronic state. We consider molecules with two electronic energy levels separated by $\Delta$. For a crystal of such molecules, the phonon branches depend therefore on the electronic states of the molecules. One can ask if that dependence does not introduce a coupling between the molecules via the optical and the acoustic branches.

It is known that for a one-dimensional chain of $N$ identical diatomic molecules there are two phonon branches, an optical branch and an acoustic one. In this study we introduce in the hamiltonian of the chain two assumptions: i) each molecule has two electronic energy levels separated by $\Delta$ and the spring constant of the spring contained in the molecule has a value which depends on its electronic state; ii) the spring constant of the spring which links two molecules nearest neighbours has a value which depends on the electronic states of both molecules linked.

One can show that phonons create on each molecule a field-like which favours the excited level and create between two molecules nearest neighbours an exchange-like interaction which can be ferro-like, antiferro-like and which can be equal to zero. For some values of $T$ and $\Delta$ the chain can display a first order phase transition with the presence of a thermal hysteresis loop. The phase transition takes place between the phase where all the molecules are in the fundamental level and that where they are in the excited one.

PACS 63.20.Kr Phonon-electron and phonon-phonon interactions- 63.50.+x Vibrational states in disordered systems- 64.60.-i General studies of phase transitions 


\section{INTRODUCTION}

In the adiabatic approximation the values of the spring constants of the springs contained in a molecule depend on its electronic state. We consider molecules with two electronic energy levels separated by $\Delta$, which is of the order of $300 K$. Such molecules can be found in various complexes of transition metal ions[1]. We therefore can assume that the values of the spring constants of the springs contained in one molecule are not the same in both levels. Consequently, in the solid state, for a crystal of $N$ identical molecules, the phonon branches

depend on the electronic states of the molecules, that is on $n_{e x}$, the fraction of molecules in the excited level. One can ask if that dependence does not introduce a coupling between the molecules via the optical and the acoustic branches. It seems clear that the optical branches which do not display a dispersion cannot contribute to such a coupling. Indeed, they correspond to springs well localized inside the molecules. In this study we consider the case of an optical branch which displays dispersion and the case of an acoustic branch.

The study of the thermal variation of $n_{e x}$ gives informations on the presence or not of a coupling. If there is not a coupling, the distance between the two energy levels is constant and the thermal variation of $n_{e x}$ is well known. For the following, we call $(a)$ and $(b)$ the fondamental and the excited electronic levels, respectivement. So, $n_{e x}$ is replaced by $n_{b}$. The levels $(a)$ and $(b)$ are obtained by quantum mechanics calculations.

It is known that for a one-dimensional chain of $N$ identical diatomic molecules there are two phonon branches, an optical branch and an acoustic one. Those results are obtained with the following assumptions: the molecules axes are parallel to the chain, the chain is periodic at the equilibrium, the atoms displacements from their equilibrim positions are longitudinal, along the chain, there is a spring between the two atoms of a molecule and one between two molecules nearest neighbours.

In this article, we study the previous linear chain with the following supplementary assumptions:

i) each molecule has two electronic energy levels separated by $\Delta$ and the spring constant of the spring contained in the molecule has a value which depends on its electronic state. This value is $k$ when the molecule is in the $(a)$ level and $\widetilde{k}$ when it is in the $(b)$ level .

ii) the spring constant of the spring which links two molecules nearest neighbours has three values: $\lambda$ when they are both in the $(a)$ level,$\nu$ when they are both in the $(b)$ level 
and $\mu$ when they are not in the same electronic level.

Ronayne et al. [2] have studied the frequency values of the normal modes of vibration of the complex $\left[\mathrm{Fe}(\text { phen })_{2}(\mathrm{NCS})_{2}\right]$ which has two electronic energy levels. They found that the majority of the frequency values are lower in the excited level than in the fundamental one. Studying the heat capacity of a crystal of this molecule, Sorai and Seki [3] have concluded that the excitation of phonons is much easier when the crystal is in the $(b)$ phase than when it is in the $(a)$ phase. In the thermodynamic $(b)$ phase ( resp. $(a)$ ) all the molecules ( or the majority of them) are in the (b) level (resp. $(a)$ ).

Following those results we assume that

$$
k \geq \widetilde{k}
$$

and

$$
\lambda \geq \mu \geq \nu
$$

The case where the values $k$ and $\widetilde{k}$ are equal while the values $\lambda, \mu$ and $\nu$ verify relation (2) looks like the case studied by Nasser [4] who has found a coupling between the molecules. So, one of aims of this study is to see if there is a coupling when the values $\lambda, \mu$ and $\nu$ are equal while the values $k$ and $\widetilde{k}$ verify relation (1).

The present study has never been done before.

The free energy of an harmonic oscillator is

$$
F=k_{B} T \ln \left(2 \sinh \left(\beta \frac{\hbar \omega}{2}\right)\right.
$$

where $\beta=\frac{1}{k_{B} T}$ and $k_{B}$ is the Boltzmann constant. It is clear that a decrease in $\omega$, the oscillator frequency value, at constant temperature lows the value of the free energy. From this result, one can say that the assumptions done in relations (1) and (2) imply that phonons favour the $(b)$ level. It is clear that the electronic parameter $\Delta$ favours the $(a)$ level . So, there is a competition between the phonons and the electronic interactions. This competition can lead to a first order phase transition. It is worth to emphasize that the lattice vibrations of the crystal have to be studied by using quantum statistical mechanics[5].

In Section 2 we present the chain hamiltonian and the variational method used to study it. In Section 3 we give the results obtained by numerical calculations and the last Section is devoted to the conclusion. 


\section{THEORETICAL STUDY}

\section{A. The Model and the Chain Hamiltonian}

Let us consider a linear chain of $N$ identical molecules each having two atoms $A$ and $B$. The molecules are numbered along the chain from left to right. The ith molecule is called $A_{i} B_{i}, i=1$ to $N$. The axes of the molecules are parallel to the chain. At equilibrium the distance between $A_{i}$ and $B_{i}$ is $d_{1}$ and that between $B_{i}$ and $A_{i+1}$ is $d_{2}$. So the chain is periodic with the period $d$ given by

$$
d=d_{1}+d_{2}
$$

The atoms $A_{i}$ and $B_{i}$ are linked by a spring with spring constant $k_{i}$, and the atoms $B_{i}$ and $A_{i+1}$ are linked by a spring with spring constant $e_{i, i+1}$. When these atoms are displaced longitudinally along the chain from they equilibrium positions by an amount $u_{i}$ for the atoms $A_{i}$ and $w_{i}$ for the atoms $B_{i}$ the potential energy of the chain is

$$
E_{p}=\sum_{i=1, . . N} \frac{1}{2} k_{i}\left(u_{i}-w_{i}\right)^{2}+\sum_{i=1, . . N} \frac{1}{2} e_{i, i+1}\left(u_{i+1}-w_{i}\right)^{2}
$$

where the sum is over the $N$ molecules. We assume periodic conditions.

The vibrations hamiltonian of the chain is

$$
H_{p h}=E_{c}+E_{p}
$$

where $E_{c}$ is the total kinetic energy of the chain.

Now we introduce the following assumptions:

i) each molecule has two electronic energy levels : a fundamental level, called $(a)$, with a degeneracy equal to the unit and an excited one, called (b), with a degeneracy equal to $r$. The separation between both levels is called $\Delta$. So, the electronic hamiltonian of the molecule $i$, can be written

$$
H_{e i}=\frac{\Delta}{2} \widehat{\sigma}_{i}
$$

where $\widehat{\sigma}_{i}$ is a fictitious spin associated to the molecule $i$ which has two eigenvalues \pm 1 , the eigenvalue -1 (resp. +1 ) corresponding to the electronic level $(a)$ ( resp. $(b)$ ). For the chain, the electronic hamiltonian is given by

$$
H_{e}=\sum_{i=1, . . N} \frac{\Delta}{2} \widehat{\sigma}_{i}
$$


ii) we assume that the spring constant $k_{i}$ has the value $k$ when the molecule $A_{i} B_{i}$ is in the $(a)$ level and the value $\widetilde{k}$ when it is in the $(b)$ level. This assumption can be written

$$
k_{i}=\frac{k+\widetilde{k}}{2}+\frac{\widetilde{k}-k}{2} \widehat{\sigma}_{i}
$$

iii) we assume that the spring constant $e_{i, i+1}$ is equal to $\lambda$ when the molecules $A_{i} B_{i}$ and $A_{i+1} B_{i+1}$ are both in the $(a)$ level, to $\nu$ when they are both in the $(b)$ level and to $\mu$ when they are not in the same level. Those assumptions can be written

$$
e_{i, i+1}=\frac{\lambda+2 \mu+\nu}{4}+\frac{\nu-\lambda}{4}\left(\widehat{\sigma}_{i}+\widehat{\sigma}_{i+1}\right)+\frac{\lambda-2 \mu+\nu}{4} \widehat{\sigma}_{i} \widehat{\sigma}_{i+1}
$$

The chain hamiltonian is

$$
H=H_{e}+H_{p h}
$$

\section{B. Phonons-molecules interactions}

By inserting equations (9) and (10) in the right hand of equation (5) the chain potential energy can be decomposed into three terms

$$
E_{p}=V_{0}+V_{1}+V_{2}
$$

with

$$
\begin{gathered}
V_{0}=\sum_{i=1, . . N} \frac{1}{2} \frac{k+\widetilde{k}}{2}\left(u_{i}-w_{i}\right)^{2}+\sum_{i=1, . . N} \frac{1}{2} \frac{\lambda+2 \mu+\nu}{4}\left(u_{i+1}-w_{i}\right)^{2} \\
V_{1}=\sum_{i=1, . . N} \frac{1}{2} \frac{\widetilde{k}-k}{2}\left(u_{i}-w_{i}\right)^{2} \widehat{\sigma}_{i}+\sum_{i=1, . . N} \frac{1}{2} \frac{\nu-\lambda}{4}\left(u_{i+1}-w_{i}\right)^{2}\left(\widehat{\sigma}_{i}+\widehat{\sigma}_{i+1}\right) \\
V_{2}=\sum_{i=1, . . N} \frac{1}{2} \frac{\lambda-2 \mu+\nu}{4}\left(u_{i+1}-w_{i}\right)^{2} \widehat{\sigma}_{i} \widehat{\sigma}_{i+1}
\end{gathered}
$$

\section{Zeeman-like interaction}

The energy term $V_{1}$ corresponds to a Zeemann-like interaction. The fields-like acting on the spin $\widehat{\sigma}_{i}$ are

$$
h_{\text {imol }}=\frac{1}{2} \frac{\widetilde{k}-k}{2}\left(u_{i}-w_{i}\right)^{2}
$$

and

$$
h_{\text {ilat }}=\frac{1}{2} \frac{\nu-\lambda}{4}\left(\left(u_{i+1}-w_{i}\right)^{2}+\left(u_{i}-w_{i-1}\right)^{2}\right)
$$


Both fields-like depend on the time, on the temperature and they are not uniform. They have the same sign and favour the $(b)$ level, that is $\widehat{\sigma}_{i}=+1$, because the coefficients $(\widetilde{k}-k)$ and $(\nu-\lambda)$ are negative (here the Zeemann-like term is written $h_{i} \widehat{\sigma}_{i}$ ).

The field-like $h_{\text {imol }}$ comes from the spring contained in the molecule $i$. But the parameter $\left(u_{i}-w_{i}\right)^{2}$ depends on the molecules $i-1, i$ and $i+1$. The field-like $h_{\text {ilat }}$ comes the springs which link the molecule $i$ with the molecules $i \pm 1$.

\section{Exchange-like interaction}

The term $V_{2}$ corresponds to an exchange-like interaction. The exchange- like constant is

$$
J_{i i+1}=\frac{1}{2} \frac{\lambda-2 \mu+\nu}{4}\left(u_{i+1}-w_{i}\right)^{2}
$$

This interaction comes from the spring which links the molecules $i$ and $i+1$. It depends on the time, on the temperature and it is not uniform. The exchange-like constant is equal to zero when

$$
\mu=\frac{\lambda+\nu}{2}
$$

It corresponds to an antiferro-like parameter when

$$
\lambda-2 \mu+\nu>0
$$

Indeed, in that case, the exchange-like interaction favours the situation where two molecules nearest neighbors are not in the same electronic state $\left(\widehat{\sigma}_{i} \widehat{\sigma}_{i+1}=-1\right)$.

The exchange-like parameter is a ferro-like one when

$$
\lambda-2 \mu+\nu<0
$$

Indeed, in that case, the exchange-like interaction favours the situation where two molecules nearest neighbors are in the same electronic state $\left(\widehat{\sigma}_{i} \widehat{\sigma}_{i+1}=1\right)$.

In this study the three previous parameters are replaced by parameters which do not depend on time and which are uniform. 


\section{Variational Method: Effective Parameters}

\section{Self-Consistent Equation}

We apply now a variational method [6] and to this end we introduce three parameters, $h, K$ and $E$. The first one $h$ describes an uniform, effective field, the second one $K$ is an effective spring constant that replaces the spring constants $k_{i}$ and the last one $E$ is an effective spring constant that replaces the spring constants $e_{i, i+1}$. Those $K$ and $E$ do not depend on the electronic states of the molecules. The variational hamiltonian $H_{0}$ is

$$
H_{0}=H_{0 s p}(h)+H_{0 p h}(K, E)
$$

The spin hamiltonian $H_{0 s p}(h)$ is

$$
H_{0 s p}(h)=\sum_{i=1, . . N}-h \widehat{\sigma}_{i}
$$

With this spin hamiltonian all the fictitious spins $\widehat{\sigma}_{i}$ have the same mean value $m$ given by

$$
m=\frac{-\exp (-\beta h)+r \exp (\beta h)}{\exp (-\beta h)+r \exp (\beta h)}
$$

Equation (24) is called self-consistent equation. The free energy related to $H_{0 s p}(h)$ is

$$
F_{0 s p}=-N k_{B} T \ln z_{0 s p}
$$

where the partition function $z_{0 s p}$ is given by

$$
z_{0 s p}=\exp (-\beta h)+r \exp (\beta h)
$$

The phonon hamiltonian $H_{0 p h}(K, E)$ is given by

$$
H_{0 p h}(K, E)=E_{c}+E_{p 0}(K, E)
$$

where

$$
E_{p 0}(K, E)=\sum_{i=1, . . N} \frac{1}{2} K\left(u_{i}-w_{i}\right)^{2}+\sum_{i=1, . . N} \frac{1}{2} E\left(u_{i+1}-w_{i}\right)^{2}
$$

The hamiltonian $H_{0 p h}(K, E)$, is the phonon hamiltonian of a linear chain of $N$ identical diatomic molecules, $A_{i} B_{i}$ with $i=1, . ., N$. The atoms $A_{i}$ and $B_{i}$ are linked by a spring with 
spring constant $K$ and two molecules nearest neighbors are linked by a spring with spring constant $E$. It is known that there are two dispersion relations

$$
\begin{aligned}
& \omega=\omega_{1}(\vec{q}) \\
& \omega=\omega_{2}(\vec{q})
\end{aligned}
$$

which correspond to the optical branch and to the acoustic branch of the chain. In the previous relation the vector $\vec{q}$ is the phonon wave vector. The previous dispersion relations are given in the Appendix.

With the hamiltonian $H_{0 p h}(K, E)$, the mean values $\left\langle\left(u_{i}-w_{i}\right)^{2}\right\rangle$ and $\left\langle\left(u_{i+1}-w_{i}\right)^{2}\right\rangle$, calculated with the matrix density of $H_{0 p h}(K, E)$, are independent on $i$ and are equal to $\left\langle\left(u_{i}-w_{i}\right)^{2}\right\rangle_{0}$ and $\left\langle\left(u_{i+1}-w_{i}\right)^{2}\right\rangle_{0}$, respectively. We have

$$
\left\langle\left(u_{i}-w_{i}\right)^{2}\right\rangle_{0}=\frac{2}{N} \sum_{\vec{q}}^{\prime} \operatorname{coth} \beta \frac{\hbar \omega(\vec{q})}{2} \frac{\hbar}{2} \frac{\partial \omega}{\partial K}
$$

and

$$
\left\langle\left(u_{i+1}-w_{i}\right)^{2}\right\rangle_{0}=\frac{2}{N} \sum_{\vec{q}}^{\prime} \operatorname{coth} \beta \frac{\hbar \omega(\vec{q})}{2} \frac{\hbar}{2} \frac{\partial \omega}{\partial E}
$$

where $\sum_{\vec{q}}^{\prime}$ is the sum over the two phonon branches and $\frac{\partial \omega}{\partial K}$ and $\frac{\partial \omega}{\partial E}$ are the partial derivatives versus $K$ and $E$, respectively, of the dispersion relations $\omega_{1}(\vec{q})$ and $\omega_{2}(\vec{q})$. The expressions of the partial derivatives are given in the Appendix. The free energy related to $H_{0 p h}(K, E)$ is

$$
F_{0 p h}=k_{B} T \sum_{\vec{q}}^{\prime} \ln \left(2 \sinh \beta \frac{\hbar \omega(\vec{q})}{2}\right)
$$

The free energy associated to the variational Hamiltonian $H_{0}$ is

$$
F_{0}=F_{0 s p}+F_{0 p h}
$$

At the first order perturbation calculation,

$$
\begin{gathered}
K=\frac{k+\widetilde{k}}{2}+\frac{\widetilde{k}-k}{2} m \\
E=\frac{\lambda+2 \mu+\nu}{4}+\frac{\nu-\lambda}{4} 2 m+\frac{\lambda-2 \mu+\nu}{4} m^{2}
\end{gathered}
$$

and 


$$
h=-\frac{\Delta}{2}+h_{p h}
$$

with

$$
h_{p h}=h_{p h m o l}+h_{p h l a t}
$$

where

$$
h_{p h m o l}=\frac{1}{2} \frac{k-\widetilde{k}}{2}\left\langle\left(u_{i}-w_{i}\right)^{2}\right\rangle_{0}
$$

and

$$
h_{\text {phlat }}=\left(\frac{\lambda-\nu}{4}-\frac{\lambda-2 \mu+\nu}{4} m\right)\left\langle\left(u_{i+1}-w_{i}\right)^{2}\right\rangle_{0}
$$

The crystal free energy corresponding to the approximation done in this study, is

$$
F=F_{0}+N\left(\frac{\Delta}{2}+h\right) m
$$

The (b) level fraction is given by

$$
n_{b}=\frac{1+m}{2}
$$

It increases from 0 to 1 when the parameter $m$ varies from -1 to 1 .

The sound velocity of longitudinal waves is

$$
V_{s}=b \sqrt{\frac{K E}{K+E} \frac{1}{m_{A}+m_{B}}}
$$

\section{Discussion}

i) We have verified that the value of the spring constant $E$ is always positive. It decreases from $\lambda$ to $\nu$ when $m$ increases from -1 to 1 .

ii) From relation (28), the thermal mean value of the potential energy $E_{p 0}(K, E)$ is given by

$$
\left\langle E_{p 0}(K, E)\right\rangle_{0}=\sum_{i=1, . . N} \frac{1}{2} K\left\langle\left(u_{i}-w_{i}\right)^{2}\right\rangle_{0}+\sum_{i=1, . . N} \frac{1}{2} E\left\langle\left(u_{i+1}-w_{i}\right)^{2}\right\rangle_{0}
$$


By inserting equations (29) and (30) in equation (42), we can verify the virial theorem

$$
\left\langle E_{p 0}(K, E)\right\rangle_{0}=\frac{1}{2}\left\langle H_{0 p h}(K, E)\right\rangle_{0}
$$

iii) In relation (38) the exchange-like interaction is studied as a mean field approximation. This study method could be improved for a $1 D$ system by using the transfert matrix method, if it is possible. In the antiferro-like case it could be interesting to introduce two sublattices.

We assume for relation (38) that the exchange-like interaction is small compared to the Zeemann-like interaction, that is

$$
|\lambda-2 \mu+\nu|<<\lambda-\nu
$$

where $|\lambda-2 \mu+\nu|$ is the absolute value of the parameter $\lambda-2 \mu+\nu$. With this condition the parameter $h_{\text {phlat }}$ is always positive.

iiii) It is worth to notice that, in the expression of the uniform field $h$, the electronic term $-\Delta$ is negative while the phonon term $h_{p h}$ is positive.

\section{NUMERICAL STUDY}

The numerical study consists essentially in solving the self-consistent equation. For that, it interesting to use reduced parameters.

\section{A. Reduced Parameters}

We take $\lambda$ as the unit of elastic force constant and $\hbar \omega_{M}(\lambda)$ as the unit of energy with

$$
\omega_{M}(\lambda)=2 \sqrt{\frac{\lambda}{m_{A}+m_{B}}}
$$

The value of $\hbar \omega_{M}(\lambda)$ is roughly estimated to $1000 \mathrm{~K}$ or $695 \mathrm{~cm}^{-1}[5]$.

We introduce the following reduced parameters:

reduced temperature

$$
t=\frac{k_{B} T}{\hbar \omega_{M}(\lambda)}
$$

the reduced electronic energy gap

$$
\delta=\frac{\Delta}{\hbar \omega_{M}(\lambda)}
$$


the elastic force constants ratios

$$
\begin{gathered}
x=\frac{\nu}{\lambda} \\
\text { xint }=\frac{\widetilde{k}}{k}
\end{gathered}
$$

and

$$
z=\frac{k}{\lambda}
$$

From condition (1)

$$
0 \leq x i n t \leq 1
$$

and from condition (2)

$$
0 \leq x \leq 1
$$

It is interesting to introduce the parameter $y$ by the relation

$$
\mu=\frac{\lambda+v}{2}+y \frac{\lambda-v}{2}
$$

The value $\mu$ varies from $\nu$ to $\lambda$ when $y$ varies from -1 to +1 . For $y=0$ the exchange-like parameter ( see $V_{2}$ ) is equal to zero. It is an antiferro-like parameter when $y<0$ and a ferro-like one when $y>0$.

Using relation (53) we have

$$
\lambda-2 \mu+\nu=-y(\lambda-\nu)
$$

So condition (44) can be written

$$
\frac{|\lambda-2 \mu+\nu|}{\lambda-\nu}=|-y|<<1
$$

\section{B. Values of the Ratio $m_{B} / m_{A}$}

We assume that $m_{A}+m_{B}$, the mass of the molecule, is $500 \mathrm{~g} \mathrm{~mol}^{-1}[7]$. So, the ratio $m_{B} / m_{A}$ is near 0.002 if the atom $B$ is an Hydrogen atom, and to 0.02 if the atom $B$ is a Carbone or a Nitrogen one.

The good reduced parameter to use for studying the self-consistent equation is

$$
p=\frac{m_{R}}{m_{A}+m_{B}}
$$


where $m_{R}$ is the reduced mass (see Appendix). The parameter $p$ is nearly equal to the ratio $m_{B} / m_{A}$. In the following, we have studied both cases $p=0.002$ and $p=0.02$. The parameter $p$ varies but the molecular mass is constant.

\section{Study of the Self-Consistent Equation}

We fix the values of all the model parameters and we look for the values $m$ which satisfy equation (24). For each solution of value $m$, we can calculate the free energy, the $(b)$ level fraction and the chain sound velocity by using relations (39), (40) and (41), respectively.

The self-consistent equation leads to either one or else three solutions. When there is only one solution, the chain is in the $(b)$ phase if the value of $n_{b}$ is near the unit and in the (a) phase if this value is near zero.

When there are three solutions, the values of the free energy of the three solutions must be compared. The thermodynamic stable solution is the one with the lowest associated free energy. When the values of the free energy of two solutions are equal both solutions are stable and the chain shows a first order phase transition. In that case, the third solution corresponds to an unstable state. When there are three solutions, the value of $t$ is the value of the transition temperature and that of $\delta$ is the value of $\delta$ at the transition.

The two solutions which correspond to the same free energy are not equal. Let us call $m_{b}$ the higher value and $m_{a}$ the other. The discontinuity in $m$ at the transition is defined by $\Delta m=m_{b}-m_{a}$. It is known that the magnitude of this discontinuity decreases as the transition temperature increases and that it disappears at the critical temperature $t_{C}$. We have used this property for finding the values of the critical point coordinates. In fact it is difficult to obtain near the critical point, the three solutions of the self-consistent equation. In this study, we consider that the critical point is reached when the discontinuity in $m$ is lower than 0.210 . So, the values of $\delta_{C}$ and $t_{C}$ obtained in this study are somewhat smaller than the exact values.

We have studied for different values of the model parameters the phase diagram of the chain, the thermal variation of the $(b)$ level fraction and that of the chain sound velocity.

For each solution, we have calculated numerically the ratio $\frac{d F}{d m}$ where $d F$ is the variation of the free energy associated to $d m$, a small variation of $m$. We have verified that this ratio 
is positive for all the solutions except the one previously called unstable solution.

We recall that, in the theory of the first order phase transition, the unstable solution plays role in the thermal hysteresis loop which appears around the transition temperature.

\section{Results}

In this study the lenght of the chain is $N=2000$ and the degeneracy of the excited level is $r=5$.

\section{Case $\lambda=\mu=\nu$.}

In that case the field-like $h_{\text {phlat }}$ and the exchange-like interaction are equal to zero. Only the field-like $h_{\text {phmol }}$ created by the springs contained in the molecules plays role. This case has never been studied before.

Relation (37) can be written

$$
h_{\text {phmol }}=\lambda \frac{z}{2} \frac{(1-x i n t)}{2}\left\langle\left(u_{i}-w_{i}\right)^{2}\right\rangle_{0}
$$

From relation (57) the field-like $h_{\text {phmol }}$ depends on the parameters xint and $z$, and also

on the mean value $\left\langle\left(u_{i}-w_{i}\right)^{2}\right\rangle_{0}$. This mean value depends on the spring constants $E$ and $K$ which depend on all the model parameters.

The chain phase diagram for xint $=0.4$ is shown in Figure 1 . The coordinates of the points of Figure 1 are the chain transition temperature and the value of $\delta$ at the transition. The (b) phase - $(a)$ phase coexistence curve is ended by the critical point $C$ with the coordinates $\left(\delta_{C}, t_{C}\right)$. Its slope is positive in agreement with the Clapeyron equation [4]. The $(a)$ phase is stable in the region above the coexistence curve and the $(b)$ phase is stable below it.

The experimental study of the first order phase transitions and that of the related effects cannot be done if the value of the critical temperature is too small. For this reason we have studied the variations of the reduced critical temperature with the model parameters. The variations of $t_{C}$ with xint is shown in Figure 2. For $p=0.002$ the atom $B$ is an Hydrogen atom, for $p=0.02$ it is a Carbone or a Nitrogen one. As shown in Figure 2, the $t_{C}$ value increases when xint decreases and it is divided by nearly 2.4 when the mass of the atom $B$ is multiplied by 10 . 
The variations of $t_{C}$ with the parameter $z$ is shown Figure 3. In this Figure xint $=0.4$. The value of the ratio $\frac{\Delta t_{C}}{\Delta z}$ is near 0.09 for $p=0.002$ and near 0.03 for $p=0.02$. In previous ratio, $\Delta t_{C}$ is the variation of $t_{C}$ for the variation $\Delta z$ of $z$.

The thermal variation of $n_{b}$ is shown in Figure 4 . For curve $(1), \widetilde{k}=k$. So the field-like $h_{p h m o l}$ is equal to zero and the effective field $h$ is equal to $-\Delta$. There is not an interaction between molecules. From Eq.(24), we deduce that $m=0$ when $t \ln (r)=\delta$. In that case $n_{b}$ is equal to 0.5 . With $r=5$ and $\delta=1.445$, the (b) level fraction is equal to 0.5 for $t=0.9$, as shown in Figure 4 . For curves $(2)$ and $(3), \widetilde{k}=0.4 k$. So the field-like $h_{\text {phmol }}$ is not equal to zero and it is the same in the curves (2) and (3). The presence or not of a discontinuity depends on the value of $\delta$ compared to the critical value $\delta_{C}=1.454$. In curve (2) the value of $\delta$ being lower than $\delta_{C}$, there is a discontinuity; in curve (3) this value being higher than $\delta_{C}$, there is not a discontinuity.

The thermal variation of the sound velocity is shown in Figure 5. The sound velocity varies when the spring constants $E$ and $K$ vary. The discontinuity of the sound velocity in the curve (2) corresponds to that of $n_{b}$ in the curve (2) of Figure 4. As shown in Figure 5, the value of the sound velocity is the same for the three curves at $0 K$. This result is due to the fact that, at $0 K$, all the molecules of the chain are in the fundamental level for the three curves, and consequently, the values of the spring constants $E$ and $K$ are the same for the three curves.

The $(b)$ level fraction and the sound velocity display an hysteresis loop around the transition temperature. The hysteresis loop of the sound velocity is shown in Figure 6. In this Figure, heating the chain from the $(a)$ phase, its temperature increases and it passes successively in the different states of the $(a)$ phase which are stable. At the transition temperature value, $t_{t}$, the chain stays in the $(a)$ phase which is now metastable. It can stay in this phase up to the temperature value $t_{u p}$ at which it is forced to pass in the $(b)$ phase which is the stable one. In fact, the chain can pass in the stable phase at any temperature comprised between $t_{t}$ and $t_{u p}$. Cooling the chain from the $(b)$ phase we find similar behaviour.

$$
\text { 2. } C a s e h_{p h m p l} \neq 0 \text { and } h_{\text {phlat }} \neq 0
$$


In that case the field-like created by phonons is the sum of the two field-like $h_{p h m o l}$ and $h_{\text {phlat }}$.

Using the reduced parameters and relation (54) in relation (38) the field-like $h_{\text {phlat }}$ can be written

$$
h_{\text {phlat }}=\frac{\lambda}{4}(1-x)(1+y m)\left\langle\left(u_{i+1}-w_{i}\right)^{2}\right\rangle_{0}
$$

This field-like depends on the parameters $x, y$ and on the mean value $\left\langle\left(u_{i+1}-w_{i}\right)^{2}\right\rangle_{0}$. This mean value depends on all the model parameters.

We have verified that the value of the critical temperature due to both fields-like is higher than the sum of the values due to each field-like. For example: for $(\lambda=\mu=\nu$; $z=1, p=0.002$, xint $=0.4)$ the value of the critical temperature is $t_{C}(\mathrm{~mol})=0.07175$. This value est due to the field-like $h_{\text {phmol }}$ alone. For $(x=0.6, y=0 ; z=1, p=0.002$, xint $=1)$ the value of the critical temperature is $t_{C}(l a t)=0.02758$. This value est due to the field-like $h_{\text {phlat }}$ alone. For $(x=0.6, y=0 ; z=1, p=0.002$, xint $=0.4)$ both field-like are different of zero and the value of the chain critical temperature is 0.22067 . This value is higher than the sum $t_{C}(\mathrm{~mol})+t_{C}(l a t)=0.09933$. We can say that the presence of the two fields-like amplifies the value of the chain critical temperature.

The role of the parameter $y$ is shown in Figure 7 . The coordinates $\left(\delta_{C}, t_{C}\right)$ of the critical point vary with the value of $y$.

\section{CONCLUSIONS}

In this study we have considered two kinds of springs: the springs which link the atoms $A_{i}$ and $B_{i}$ of the molecule $A_{i} B_{i}, i=1, . . N$, and the springs which link two molecules first neighbours along the chain. We have first studied the case where only the spring constants of the springs contained in the molecules depend on the electronic state of the molecules. We have shown that, in this case, phonons create on each molecule a field-like which favours the excited level, or $(b)$ level. The competition between this field-like and the electronic parameter $\Delta$, which favours the fundamental level, or $(a)$ level, leads, for some values of $T$ and $\Delta$, to a first order phase transition. This result is important because it is certainly possible to identify in a molecule which springs contained in the molecule can contribute, in the solid state, to the coupling. 
We have also studied, in this case, the variations of the critical temperature value with the model parameters. This study is necessary because the first order phase transition and the related effects cannot be observed if the value of the critical temperature is too small. One interesting result is that the value of the critical temperature is three time higher when the atom $B$ is an Hydrogen atom than when it is a Carbon or Nitrogen one. This result leads to think that hydrogen bonds can play role in the coupling between the molecules in the solid state.

We have then added the assumption that the spring constants of the springs which link two molecules first neighbours depend on the electronic states of both molecules linked. In that case, phonons create un new field-like which also favours the $(b)$ level and also create an exchange-like interaction between molecules first neighbours. The interesting result is that the presence of both fields-like increases a lot the critical temperature value.

Some studies $[5,8,9]$ have been done with the assumption that only the spring constants of the springs which link two molecules first neighbours depend on the electronic states of both molecules linked. In those studies the authors have to take small values, near 0.2 , for the ratio $\frac{\nu}{\lambda}$ in order to have a critical temperature value not too small. We recall that $\lambda$ is the value of the spring constant when both linked molecules are in the $(a)$ level and $\nu$ is this value when they are both in the $(b)$ level. We think that the same studies could be done with higher value for the ratio $\frac{\nu}{\lambda}$ if both fields-like are introduced in the model.

Some molecules which have two electronic energy levels are organised around an iron II ion in an octahedral environment. The value of the spin total of the $3 d$ electrons is $S=0$ for the fundamental level and $S=2$ for the excited one. When the molecule passes from the fundamental level to the excited one the value of its spin changes. For this reason it is called a spin conversion molecule. A crystal of $N$ identical spin conversion molecules is called a spin conversion compound. For some spin conversion compounds, the thermal variation of the fraction of molecules in the excited level displays an hysteresis loop which means that there is a coupling between the molecules in the solid state. Many models have been proposed for the origin of this coupling[10 - 14,4]. We propose the present model which has three characteristics: it is founded on the adiabatic approximation which is a well admitted approximation, experimental studies and DFT calculations show that the values of the spring constants of the springs contained in the molecule vary with its electronic state $[2,15-17]$ and the exchange-like interaction allows to reproduce many experimental 
results obtained on spin conversion compounds[8, 9].

\section{APPENDIX}

\section{A. Phonon dispersion relations}

We consider consider a chain of $2 N$ atoms with $N$ units cell but with two atoms in each cell so there are $2 N$ atoms. At the equilibrium the period of the translational symmetry is $b$. For two atoms per cell we find two dispersion curves. The acoustic branch has a frequency equal to zero when the wave vector $\vec{q}$ is equal to zero, while the optic branch has a finite frequency for this value of $\vec{q}$. The atoms are displaced longitudinally along the chain from their equilibrium positions.

The dispersion relation for the optic branch is

$$
\omega_{o p}^{2}=\frac{K+E}{2 m_{R}}+\sqrt{\left(\frac{K+E}{2 m_{R}}\right)^{2}-\frac{4 K E}{m_{A} m_{B}} \sin ^{2} \frac{q b}{2}}
$$

and that for the acoustic branch is

$$
\omega_{a c}^{2}=\frac{K+E}{2 m_{R}}-\sqrt{\left(\frac{K+E}{2 m_{R}}\right)^{2}-\frac{4 K E}{m_{A} m_{B}} \sin ^{2} \frac{q b}{2}}
$$

In the above relations, $m_{R}$ is the reduced mass

$$
m_{R}=\frac{m_{A} m_{B}}{m_{A}+m_{B}}
$$

Using the periodic conditions the wave vector $q$ verifies the relation

$$
q b=n_{p h} \frac{2 \pi}{N}
$$

with

$$
n_{p h}=0, \pm 1, \pm 2, \ldots, \frac{N}{2} \text { when } N \text { is even }
$$

When $q \rightarrow 0$, the above relations become

$$
\omega_{o p}^{2} \simeq \frac{K+E}{m_{R}}+O\left(q^{2}\right)
$$

where $O\left(q^{2}\right)$ is a function of $q^{2}$ and 


$$
\omega_{a c}^{2} \simeq \frac{K E}{K+E} \frac{1}{m_{A}+m_{B}} b^{2} q^{2}=V_{s}^{2} q^{2}
$$

where $V_{s}$ is the sound velocity.

When $K \gg E$, the dispersion relations become

$$
\omega_{o p}^{2} \simeq \frac{K}{m_{R}}+O\left(q^{2}\right)
$$

and

$$
\omega_{a c}^{2} \simeq 4 \frac{E}{m_{A}+m_{B}} \sin ^{2} \frac{q b}{2}
$$

So, the acoustic frequency values are those of a linear chain of atoms with the masse $m_{A}+m_{B}$.

\section{B. Partial derivatives of $\omega$}

We introduce $a$ by

$$
\omega=\sqrt{a}
$$

with

$$
a=\frac{K+E}{2 m_{R}} \pm \sqrt{c}
$$

and

$$
c=\frac{(K+E)^{2}}{4 m_{R}^{2}}-\frac{4 K E}{m_{A} m_{B}} \sin ^{2} \frac{q b}{2}
$$

So we have

$$
\frac{\partial \omega}{\partial K}=\frac{1}{2 \omega} \frac{\partial a}{\partial K}
$$

and

$$
\frac{\partial \omega}{\partial E}=\frac{1}{2 \omega} \frac{\partial a}{\partial E}
$$

with

$$
\frac{\partial a}{\partial K}=\frac{1}{2 m_{R}} \pm \frac{1}{2 \sqrt{c}}\left(\frac{2(K+E)}{4 m_{R}^{2}}-\frac{4 E}{m_{A} m_{B}} \sin ^{2} \frac{q b}{2}\right)
$$

and

$$
\frac{\partial a}{\partial E}=\frac{1}{2 m_{R}} \pm \frac{1}{2 \sqrt{c}}\left(\frac{2(K+E)}{4 m_{R}^{2}}-\frac{4 K}{m_{A} m_{B}} \sin ^{2} \frac{q b}{2}\right)
$$

In the above relations, the symbol \pm must be replaced by + for the frequency values of the optical branch and by - for those of the acoustic one. 
So,

$$
\begin{aligned}
& \frac{\partial \omega}{\partial K}=\frac{1}{2 \omega}\left(\frac{1}{2 m_{R}} \pm \frac{1}{2 \sqrt{c}}\left(\frac{2(K+E)}{4 m_{R}^{2}}-\frac{4 E}{m_{A} m_{B}} \sin ^{2} \frac{q b}{2}\right)\right) \\
& \frac{\partial \omega}{\partial E}=\frac{1}{2 \omega}\left(\frac{1}{2 m_{R}} \pm \frac{1}{2 \sqrt{c}}\left(\frac{2(K+E)}{4 m_{R}^{2}}-\frac{4 K}{m_{A} m_{B}} \sin ^{2} \frac{q b}{2}\right)\right)
\end{aligned}
$$

Keywords: Phase transition. Lattices vibrations.

\section{REFERENCES}

1. J. Owen, J. H. Thomley, Rep. Prog. Phys. 29, 675 (1966)

2. K. L. Ronayne, H. Paulsen, A. Höfer, A. C. Dennis, J. A. Wolny, A. I. Chumakov, V. Schünemann, H. Winkler, H. Spiering, A. Bousseksou, P. Gütlich, A. X. Trautwein and J. J. McGarney, Phys. Chem. Chem. Phys. 8, 4685-4693 (2006)

3. M. Sorai and S. Seki, J. Phys.Chem.Solids. 35, 555-570 (1974)

4. J.A. Nasser Eur. Phys. J. B 21, 3 (2001)

5. J. A. Nasser, S. Topçu, L. Chassagne, M. Wakim, B. Bennali, J. Linares and Y. Alayli, Eur. Phys. J. B, vol.83, (2011) p.115

6. R. Balian, Microphysics to Macrophysics. Methods and Applications of Statistical Physics ( Berlin Heidelberg New York, Springer-Verlag, 1991)

7. J.-P. Martin, Mécanisme des Transitions de Spin dans les Composés Moléculaires à l'Etat Solide. Effet de la Dilution de l'Ion métallique,Thèse,Université de Paris-Sud, Centre d'Orsay, 1994

8. J.A. Nasser, K. Boukheddaden and J. Linares Eur. Phys. J. B 39, 219 (2004)

9. J.A. Nasser Eur. Phys. J. B 48, 19 (2005)

10. J. Wajnflasz, Phys. Stat. Solidi 40, 537 (1970)

11. R. Zimmermann, E. König, JPCS 38, 779 (1977) 
12. H. Spiering, E. Meissner, H. Köppen, E. W. Müller, P. Gütlich, Chem. Phys. Lett. 91, $348(1982)$

13. P. Gütlich Structure and Bonding ( Springer-Verlag, Berlin, 1981) Vol. 44

14. A. Bousseksou, J. Nasser, J. Linares, K. Boukheddaden and F. Varret, J. Phys. I, 2, $1381-1403(1992)$

15. Azzedine Bousseksou, Marc Verelst, Hector Constant-Machado, Gilles Lemercier, Jean-Pierre Tuchagues and François Varret, Inorg. Chem., 35, 110-115 (1996)

16. Azzedine Bousseksou, John J. McGarvey, François Varret, José Antonio Real, JeanPierre Tuchagues, Andrew C. Dennis, Marie Laure Boillot, Chemical Physics Letters 318, 409-416 (2000)

17. Villõ K. Palfi, Thomas Guillon, Hauke Paulsen, Gabor Molnãr, Azzedine Bousseksou, C. R. Chimie 8 1317-1325 (2005)

\section{Figures Captions}

Figure 1. Chain phase diagram. Only the spring constants of the springs contained in the molecules depend on the electronic state of the molecules. Phonons create a field-like which favours the $(b)$ level while the electronic parameter $\Delta$ favours the $(a)$ level. This competition leads to a first order phase transition. The coexistence curve is ended by the critical point $C$ with the coordinates $\delta_{C}=1.45404$ and $t_{C}=0.07175$. In this Figure $z=\frac{k}{\lambda}=1$, xint $=\frac{\widetilde{k}}{k}=0.4$ and $p=0.002$. For this value of the parameter $p$, the atom $B$ of the molecule $A B$ is an Hydrogen. $\widetilde{k}$

Figure 2 Variations of the chain critical temperature with the reduced parameter xint $(=$ $\left.\frac{k}{k}\right)$. In this Figure $z=1$.

Figure 3. Variations of the chain critical temperature with the ratio $z=\frac{k}{\lambda}$. In this Figure xint $=0.4$.

Figure 4. Thermal variation of the $(b)$ level fraction. In curve (1) there is not an interaction between the molecules and the distance between both energy levels is constant. 
In curves (2) and (3) there is an interaction between the molecules which leads to a discontinuity when the value of $\delta$ is lower than the critical value $\delta_{C}=1.454$. In this Figure $z=1$.

Figure 5. Thermal variation of sound velocity. The values of the model parameters are the same in Figures 4 and 5. In curve (1) the spring constants $E$ and $K$ do not vary with the temperature because there is not an interaction between the molecules. Consequently the sound velocity is constant. In curves (2) and (3) there is an interaction between the molecules which leads to a thermal variation of $E$ and $K$, and of the sound velocity. There is a discontinuity when the value of $\delta$ is lower than the critical value $\delta_{C}=1.454$.

Figure 6. Sound velocity thermal hysteresis loop. Open circle: stable state; dark circle: metastable state; cross: unstable state. The transition temperature value is between 0.0648 and 0.0650. The limits of the hysteresis loop are $t_{u p}=0.0663$ and $t_{\text {down }}=0.0595$. The loop width is $\Delta t=6.810^{-3}$. The thermodynamic states of the $(a)$ phase are stable below the transition temperature and those of the $(b)$ phase are stable above it. The values of the model parameters are the same as in Figure 4.

Figure 7. Chain phase diagrams for $y=0$. and $y= \pm 0.1$. The coordinates of the critical point vary with the $y$ values: $t_{C}$ is equal to $0.1688,0.2207$ and 0.2777 for $y$ equal to $-0.1,0$. and 0.1 , respectivement. The model parameters are $(x=0.6, y ; z=1, p=0.002, x i n t=0.4$ ) . 